\title{
Synthesis and insecticidal activity of $N$-cyano 2-(substituted amino) ethyl methyl sulfoximine derivatives
}

\author{
Haibo Yu, Zhenfang Qin, Hong Dai, Xin Zhang, Xue Qin, Tingting Wang, and \\ Jianxin Fang*
}

State Key Laboratory of Elemento-Organic Chemistry, Research Institute of Elemento-Organic

Chemistry, Nankai University, Tianjin 300071, People's Republic of China

E-mail:fjx@nankai.edu.cn

\begin{abstract}
The $N$-cyano 2-(substituted amino)ethyl methyl sulfoximine derivatives are a new chemical family of neonicotinoids. Nine alkyl sulfoximine derivatives were designed and synthesized. The synthesized compounds were identified by ${ }^{1} \mathrm{H} \mathrm{NMR},{ }^{13} \mathrm{C} \mathrm{NMR}$, IR, and elemental analysis. The preliminary bioassays indicated that some of them showed moderate insecticidal activities against Myzus persicae. The relationship between structure and biological activity was also discussed.
\end{abstract}

Keywords: Sulfoximine derivatives, synthesis, insecticidal activity, peach aphid

\section{Introduction}

Since the advent of imidacloprid $\mathbf{1}^{1} 15$ years ago, the search for new neonicotinoid insecticides has been an intense and competitive effort on the part of several research groups within the agrochemical industry. Subsequent research resulted in the commercialization of several outstanding insecticides (Figure 1), such as nitenpyram 2 (Takeda, 1995), acetamiprid 3 (Nippon Soda, 1996), thiacloprid 4 (Bayer, 2000), thiamethoxam 5 (Novartis, 1998), clothianidin 6 (Takeda, Bayer, 2002) and dinotefuran 7 (Mistsui, 2002) ${ }^{2-8}$ Neonicotinoid insecticides were used rapidly worldwide for controlling insects because of their high potency, low mammalian toxicity, broad insecticidal spectra, and good systemic properties. Neonicotinoids interacting with nicotinic acetylcholine receptors (nAChR) have a higher affinity for the insect receptor than for the mammalian ${ }^{9-12}$ and are relatively safe toward mammals and aquatic life. 
<smiles>O=[N+]([O-])/C=N/C1NCCN1Cc1ccc(Cl)nc1</smiles><smiles>CCN(Cc1ccc(Cl)nc1)/C(=C/[N+](=O)[O-])NC</smiles>

nitenpyram 2<smiles>C/C(=N\C#N)N(C)Cc1ccc(Cl)nc1</smiles>

acetamiprid $\mathbf{3}$<smiles>N#CN=C1SCCN1Cc1ccc(Cl)nc1</smiles>

thiacloprid 4<smiles>CN1COCN(Cc2cnc(Cl)s2)C1=N[N+](=O)[O-]</smiles>

thiamethoxam 5<smiles>CNC(=N[N+](=O)[O-])NCc1cnc(Cl)s1</smiles>

clothianidin 6<smiles>CNC(=N[N+](=O)[O-])NCC1CCOC1</smiles>

dinotefuran 7

Figure 1. Commercial neonicotinoids.

The development of resistance to insecticide in insect populations is a well recognized phenomenon and there are well documented cases of resistance for the major classes of insecticides. Although the neonicotinoids have proved relatively resilient to the development of resistance, high levels of resistance have been documented in field-collected populations of the whitefly, Bemisa tabaci. During the late 1990s, resistant species increased in potency with more recently-collected strains of this whitefly exhibiting more than 100-fold resistance to imidacloprid, and comparable levels of resistance to thiamethoxam and acetamiprid. ${ }^{13-15}$ Therefore, new insecticides that lack cross-resistance to currently available insecticides are imminently required.

In recent years, $N$-substituted (pyridyl)alkyl sulfoximine derivatives (8-10) were described by Dow AgroSciences. ${ }^{16-19}$ It has been reported that these compounds lack of cross-resistance on insect pests that have developed resistance to one ore more classes of insecticides including imidacloprid and other neonicotinoids. ${ }^{20}$ Encouraged by this report, we developed an idea that lengthening the bridge chain between heterocycle and pharmacophore ( $N$-cyano sulfoximines) might find new insecticides that are effective on the resistant insect populations. The thiazole group can be taken as a bioisostere of pyridine. Hence, using thiazolyl group to replace pyridyl, a series of $\mathrm{N}$-cyano 2-((substituted benzyl)(2-chloro-thiazol-5-ylmethyl)amino)ethyl methyl sulfoximine derivatives 17 were designed and synthesized. This paper describes the syntheses and bioactivities of a number of sulfoximine derivatives. The relationship between structure and biological activity is also discussed.<smiles>CS(=O)(=O)Cc1ccc(C(F)(F)F)nc1</smiles>

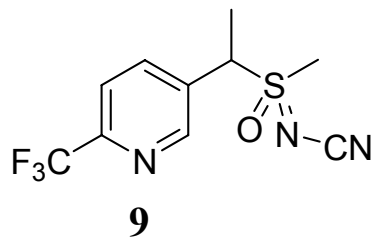

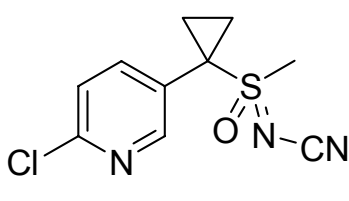

10<smiles>N#CN[SH]1(=O)CCCC1c1ccc(Cl)nc1</smiles>

11

Figure 2. Chemical structure of $N$-substituted (pyridyl)alkyl sulfoximine derivatives. 


\section{Results and Discussion}

\section{Chemistry}

The sequence of the reactions leading to the syntheses of $N$-cyano 2-(substituted amino) ethyl methyl sulfoximine derivatives in this study is outlined in Schemes 1, 2, and 3. As is shown in Scheme 1, our synthesis of title compounds was started from the thiazole. ${ }^{21}$ First, the reaction of 2-chloro-5-(chloromethylthiazole) with 2-chloroethanamine in presence of triethylamine was carried out in acetonitrile at room temperature. ${ }^{22}$ 2-Chloro- $N$-((2-chlorothiazol-5yl)methyl)ethanamine 12 was the major product. However, the formation of $10 \%$ of 5-(aziridin1-ylmethyl)-2-chlorothiazole 13 was observed (Scheme 1).

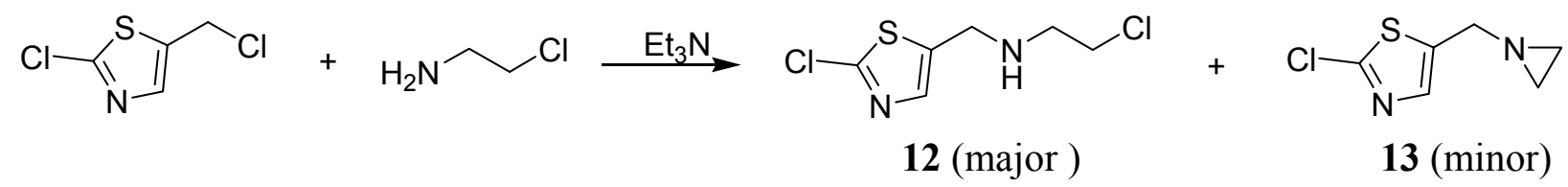

Scheme 1. Synthesis of 2-chloro-N-((2-chlorothiazol-5-yl)methyl)ethanamine.

The synthesis of sulfide $\mathbf{1 4}$ can readily be achieved by the reaction of compound $\mathbf{1 2}$ with sodium methylthiolate in good $(90 \%)$ yield. Sequentially, the compound $\mathbf{1 4}$ was subjected to reductive amination reaction to give 15 in good (68-78\%) yields. The reaction of 14 with substituted benzaldehyde in the presence reducing agents such as sodium triacetoxyborohydride was carried out in 1,2-dichloroethane. ${ }^{23}$ The synthesis of $N$-cyano sulfilimines $\mathbf{1 6}$ can readily be achieved by reaction of the corresponding sulfides with cyanogen amine in the presence of a base such as $t$-BuOK and NBS as halogenating agents. ${ }^{24}$ The solvent has a great affect on the yields, methanol provided the highest yields (Table 1). Finally, the sulfilimine was oxidized with 3-chloroperoxy-benzoid acid ( $m$-CPBA) and potassium carbonate as a base was employed to neutralize the acidity of $m$-CPBA and afforded the $N$-cyano sulfoximines 17 in good (62-75\%) yields (Scheme 3). Protic polar solvent ethanol and water were used to increase the solubility of sulfilimines starting material.

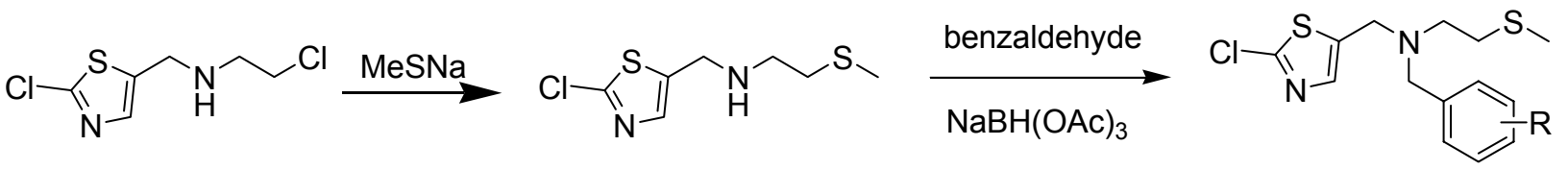

12
14
15

Scheme 2. Synthesis of $N$-substitutedbenzyl- $N$-((2-chlorothiazol-5-yl)methyl)-2-(methylthio) ethanamine. 


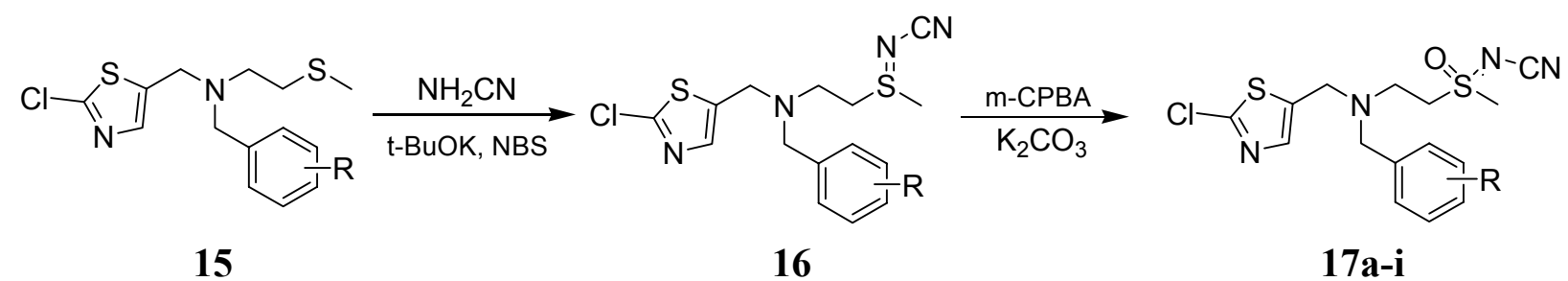

Scheme 3. Synthesis of $N$-cyano 2-(substituted amino)ethyl methyl sulfoximines.

Table 1. The different solvent effect on the yields

\begin{tabular}{ccc}
\hline Entry & Solvent & Yield of 16a $(\%)$ \\
\hline 1 & methanol & 69 \\
2 & ethanol & 55 \\
3 & tetrahydrofuran & 59 \\
4 & dichloromethane & 30 \\
5 & 1,2-dichloroethane & 40 \\
\hline
\end{tabular}

The synthesized compounds were identified by ${ }^{1} \mathrm{H}$ NMR, ${ }^{13} \mathrm{C}$ NMR, IR, and elemental analysis. In the ${ }^{1} \mathrm{H}$ NMR spectra of compound 17, a sharp peak representing the proton of the thiazole was observed in the range of 7.40-7.43 ppm. Moreover, the proton signals due to $\mathrm{SCH}_{3}$ group of these compounds were resonated in the region 3.20-3.27 ppm as a singlet integrating for the three protons. The characteristic signals resulting from the $\mathrm{C} \equiv \mathrm{N}$ group of sulfoximines were discernible at $111.78-112.03 \mathrm{ppm}$ in the ${ }^{13} \mathrm{C}$ NMR spectrum and IR spectra displayed $\mathrm{C} \equiv \mathrm{N}$ group strong absorption at $2200-2190 \mathrm{~cm}^{-1}$.

\section{Structure-activity relationships}

The newly synthesized compounds $\mathbf{1 7 a - i}$ have been tested for their insecticidal activities against peach aphid (Myzus persicae). The results of insecticidal activities are listed in Table 2. These data show that efficacy is strongly influenced by the nature of the substitutes and their position on the benzene ring. The structure-activity relationships for benzyl group were elucidated. In general, the presence of halo group or other substituted group at position 4 or 3 of phenyl ring enhanced the insecticidal activities. Adding a methyl group at the 4-position of the benzene ring led to a significant increase in activity. However, the substituted group only at 2- position of benzene ring caused a loss of the activity.

In conclusion, $\mathrm{N}$-cyano 2 -(substituted amino)ethyl methyl sulfoximine derivatives were designed and synthesized, and some title compounds exhibited moderate insecticidal activities against Myzus persicae in the concentration of $10 \mathrm{mg} / \mathrm{L}$. 
Table 2. Insecticidal activities against Myzus persicae of compounds $17 \mathrm{a}-\mathrm{i}$

\begin{tabular}{ccc}
\hline Comp No & $\mathrm{R}$ & $\begin{array}{c}\text { mortality }(\%) \text { at concentration of } \\
10 \mathrm{mg} / \mathrm{L}\end{array}$ \\
\hline $\mathbf{1 7 a}$ & $\mathrm{H}$ & 10 \\
$\mathbf{1 7 b}$ & $2-\mathrm{Cl}$ & 0 \\
$\mathbf{1 7} \mathbf{c}$ & $2-\mathrm{F}$ & 0 \\
$\mathbf{1 7 d}$ & $3-\mathrm{F}$ & 65 \\
$\mathbf{1 7}$ & $4-\mathrm{F}$ & 10 \\
$\mathbf{1 7 f}$ & $4-\mathrm{Cl}$ & 58 \\
$\mathbf{1 7 g}$ & $4-\mathrm{OMe}$ & 10 \\
$\mathbf{1 7 h}$ & $4-\mathrm{Me}$ & 75 \\
$\mathbf{1 7} \mathbf{i}$ & $2-\mathrm{F}-4-\mathrm{Br}$ & 70 \\
\hline
\end{tabular}

0 equals no activity; 100 equals total control.

\section{Experimental Section}

General Procedures. Melting points were measured by using a RY-1 melting point apparatus and are uncorrected. ${ }^{1} \mathrm{H}$ NMR and ${ }^{13} \mathrm{C}$ NMR spectra were recorded on a Varian-300 spectrometer using TMS as an internal reference. Chemical shift values $(\delta)$ were given in ppm. Infrared spectra were obtained on a Bio-Rad spectrophotometer using potassium bromide pellets or as neat oils and are reported as wave numbers $\left(\mathrm{cm}^{-1}\right)$. Mass spectra (GC-MS) were obtained on an Agilent 6890-5973 instrument. Elemental analysis was performed on a Yanaco CDRDER MT-3 elemental analyzer.

Synthesis of 2-chloro- $\mathrm{N}$-((2-chlorothiazol-5-yl)methyl)ethanamine (12). A solution of 2chloro-5-(chloromethyl)thiazole (1.68 g, $10 \mathrm{mmol}), 2$-chloroethanamine $(1.3 \mathrm{~g}, 11.2 \mathrm{mmol})$, and triethylamine $(1.53 \mathrm{~mL}, 11 \mathrm{mmol})$ in acetonitrile $(20 \mathrm{~mL})$ was stirred at $25{ }^{\circ} \mathrm{C}$ for $60 \mathrm{~h}$. The reaction was completed and the solvent was removed under reduced pressure. The residue was partitioned between dichloromethane and water, washed with saturated brine. The organic layer was dried over sodium sulfate. Then, the solvent was again removed under reduce pressure and the residue was purified by column chromatography (ethyl acetate-hexanes, 2:1 ) to yield the title compound 13 as a yellow oil (1.05 g, 50\% yield). ${ }^{1} \mathrm{H} \mathrm{NMR}\left(\mathrm{CDCl}_{3}, \delta \mathrm{ppm}\right) 2.46(\mathrm{~s}, 1 \mathrm{H}, \mathrm{NH})$, 2.98 (t, 2H, $\left.\mathrm{CH}_{2}\right), 3.67$ (s, 2H, $\left.\mathrm{CH}_{2}\right), 3.99\left(\mathrm{~s}, 2 \mathrm{H}, \mathrm{CH}_{2}\right), 7.35$ (s, 1H).

5-(Aziridin-1-ylmethyl)-2-chlorothiazole (13). Yield, 10\%, white crystaline solid, mp 135-136 ${ }^{\circ} \mathrm{C} .{ }^{1} \mathrm{H}$ NMR $\left(\mathrm{CDCl}_{3}, \delta(\mathrm{ppm})\right): 2.51\left(\mathrm{~s}, 4 \mathrm{H}, \mathrm{CH}_{2}\right), 3.65\left(\mathrm{~s}, 2 \mathrm{H}, \mathrm{CH}_{2}\right), 7.34$ (s, 1H, Thiazole).

Synthesis of $\mathbf{N}$-((2-chlorothiazol-5-yl)methyl)-2-(methylthio)ethanamine (14). A solution of 2-chloro- $N$-((2-chlorothiazol-5-yl)methyl)ethanamine $(10 \mathrm{mmol})$ in $10 \mathrm{~mL}$ of ethanol was treated with sodium methylthiolate $(10.05 \mathrm{mmol})$ at room temperature. The reaction was completed in 
$30 \mathrm{~min}$, so the solvent was removed under reduced pressure. The residue was partitioned between dichloromethane and water, washed with saturated brine and dried over sodium sulfate. The solvent was again removed under reduce pressure and the residue was purified by column chromatography (ethyl acetate-hexanes, 2:1 ) to yield the title compound $\mathbf{1 5}$ as a light yellow oil (2.0g, 90\% yield). ${ }^{1} \mathrm{H} \mathrm{NMR}\left(\mathrm{CDCl}_{3}, \delta(\mathrm{ppm})\right): 1.87(\mathrm{~s}, 1 \mathrm{H}, \mathrm{NH}), 2.08\left(\mathrm{~s}, 2 \mathrm{H}, \mathrm{CH}_{3}\right) 2.64(\mathrm{t}, 2 \mathrm{H}$, $\left.\mathrm{CH}_{2}\right), 2.83$ (s, 2H, $\left.\mathrm{CH}_{2}\right), 3.96\left(\mathrm{~s}, 2 \mathrm{H}, \mathrm{CH}_{2}\right), 7.35$ (s, 1H).

General procedure for the preparation of $N$-substitutedbenzyl- $N$-((2-chlorothiazol-5yl)methyl)-2-(methylthio)ethanamine (15)

To a solution of $N$-((2-chlorothiazol-5-yl)methyl)-2-(methylthio)ethanamine (10 mmol) in 1,2dichloroethane $(20 \mathrm{~mL})$ was added substituted benzaldehyde $(10 \mathrm{mmol})$, sodium triacetoxyborohydride $(15 \mathrm{mmol})$ and magnesium sulfate $(20 \mathrm{mmol})$. The reaction was stirred at room temperature. After consumption of the starting material (monitored by TLC) the reaction mixture was concentrated under reduced pressure. The resulting product was purified by flash column chromatography to afford the title compounds as colorless oil.

$\boldsymbol{N}$-Benzyl- $\boldsymbol{N}$-((2-chlorothiazol-5-yl)methyl)-2-(methylthio)ethanamine (15a). Yield, 75\%, colorless oil. ${ }^{1} \mathrm{H} \mathrm{NMR}\left(\mathrm{CDCl}_{3}, \delta(\mathrm{ppm})\right): 2.03$ (s, 3H, $\left.\mathrm{CH}_{3}\right), 2.59-2.74\left(\mathrm{~m}, 4 \mathrm{H}, \mathrm{CH}_{2}\right), 3.64(\mathrm{~s}, 2 \mathrm{H}$, $\left.\mathrm{CH}_{2}\right), 3.75$ (s, 2H, $\left.\mathrm{CH}_{2}\right), 7.25-7.34$ (m, 6H, Ar-H).

$\boldsymbol{N}$-(2-Chlorobenzyl)- $\mathbf{N}$-((2-chlorothiazol-5-yl)methyl)-2-(methylthio)ethanamine

(15b). Yield, 80\%, colorless oil. ${ }^{1} \mathrm{H} \mathrm{NMR}\left(\mathrm{CDCl}_{3}, \delta(\mathrm{ppm})\right)$ : $2.04\left(\mathrm{~s}, 3 \mathrm{H}, \mathrm{CH}_{3}\right), 2.63-2.77\left(\mathrm{~m}, 4 \mathrm{H}, \mathrm{CH}_{2}\right)$, 3.77 (s, 2H, $\left.\mathrm{CH}_{2}\right), 3.80$ (s, 2H, $\left.\mathrm{CH}_{2}\right), 7.08-7.12$ (m, 2H, Ar-H), 7.28-7.34 (m, 2H, Ar-H), 7.55$7.58(\mathrm{~m}, 2 \mathrm{H}, \mathrm{Ar}-\mathrm{H})$.

$\boldsymbol{N}$-(2-Fluorobenzyl)- $\boldsymbol{N}$-((2-chlorothiazol-5-yl)methyl)-2-(methylthio)ethanamine (15c). Yield, 85\%, colorless oil. ${ }^{1} \mathrm{H}$ NMR $\left(\mathrm{CDCl}_{3}, \delta(\mathrm{ppm})\right): 2.05\left(\mathrm{~s}, 3 \mathrm{H}, \mathrm{CH}_{3}\right), 2.61-2.77\left(\mathrm{~m}, 4 \mathrm{H}, \mathrm{CH}_{2}\right), 3.64$ (s, 2H, $\left.\mathrm{CH}_{2}\right), 3.76\left(\mathrm{~s}, 2 \mathrm{H}, \mathrm{CH}_{2}\right), 6.95-6.96(\mathrm{~m}, 1 \mathrm{H}, \mathrm{Ar}-\mathrm{H}), 7.08-7.12$ (m, 2H, Ar-H), 7.26-7.34 (m, $1 \mathrm{H}, \mathrm{Ar}-\mathrm{H}), 7.35$ (s, 1H, Thiazole).

$\boldsymbol{N}$-(3-Fluorobenzyl)- $\mathbf{N}$-((2-chlorothiazol-5-yl)methyl)-2-(methylthio)ethanamine (15d).Yield, 83\%, colorless oil. ${ }^{1} \mathrm{H}$ NMR $\left(\mathrm{CDCl}_{3}, \delta(\mathrm{ppm})\right): 2.04\left(\mathrm{~s}, 3 \mathrm{H}, \mathrm{CH}_{3}\right), 2.61-2.74\left(\mathrm{~m}, 4 \mathrm{H}, \mathrm{CH}_{2}\right), 3.73$ (s, 2H, $\left.\mathrm{CH}_{2}\right), 3.78$ (s, 2H, $\left.\mathrm{CH}_{2}\right), 7.00-7.06(\mathrm{~m}, 1 \mathrm{H}, \mathrm{Ar}-\mathrm{H}), 7.11-7.16(\mathrm{~m}, 1 \mathrm{H}, \mathrm{Ar}-\mathrm{H}), 7.21-7.29$ (m, 1H, Ar-H), 7.36 (s, 1H, Thiazole), 7.41-7.46 (m, 1H, Ar-H).

$\boldsymbol{N}$-(4-Fluorobenzyl)- $\mathbf{N}$-((2-chlorothiazol-5-yl)methyl)-2-(methylthio)ethanamine (15e). Yield, 75\%, colorless oil. ${ }^{1} \mathrm{H}$ NMR $\left(\mathrm{CDCl}_{3}, \delta(\mathrm{ppm})\right): 2.03\left(\mathrm{~s}, 3 \mathrm{H}, \mathrm{CH}_{3}\right), 2.60-2.74\left(\mathrm{~m}, 4 \mathrm{H}, \mathrm{CH}_{2}\right), 3.60$ (s, 2H, $\left.\mathrm{CH}_{2}\right), 3.76$ (s, 2H, $\left.\mathrm{CH}_{2}\right), 6.98-7.04$ (m, 2H, Ar-H), 7.27-7.33 (m, 2H, Ar-H), 7.34 (s, 1H, Thiazole).

$\boldsymbol{N}$-(4-Chlorobenzyl)- $\mathbf{N}$-((2-chlorothiazol-5-yl)methyl)-2-(methylthio)ethanamine (15f). Yield, 88\%, colorless oil. ${ }^{1} \mathrm{H}$ NMR $\left(\mathrm{CDCl}_{3}, \delta(\mathrm{ppm})\right): 2.04\left(\mathrm{~s}, 3 \mathrm{H}, \mathrm{CH}_{3}\right), 2.61-2.72\left(\mathrm{~m}, 4 \mathrm{H}, \mathrm{CH}_{2}\right), 3.60$ (s, 2H, $\left.\mathrm{CH}_{2}\right), 3.75$ (s, 2H, $\left.\mathrm{CH}_{2}\right), 7.26-7.34$ (m, 5H, Ar-H).

$\boldsymbol{N}$-(4-Methoxybenzyl)- $\boldsymbol{N}$-((2-chlorothiazol-5-yl)methyl)-2-(methylthio) ethanamine (15g). Yield, 79\%, colorless oil. ${ }^{1} \mathrm{H} \mathrm{NMR}\left(\mathrm{CDCl}_{3}, \delta(\mathrm{ppm})\right)$ : $2.03\left(\mathrm{~s}, 3 \mathrm{H}, \mathrm{CH}_{3}\right), 2.59-2.72\left(\mathrm{~m}, 4 \mathrm{H}, \mathrm{CH}_{2}\right)$, 
$3.58\left(\mathrm{~s}, 2 \mathrm{H}, \mathrm{CH}_{2}\right), 3.74\left(\mathrm{~s}, 2 \mathrm{H}, \mathrm{CH}_{2}\right), 3.79\left(\mathrm{~s}, 3 \mathrm{H}, \mathrm{OCH}_{3}\right), 6.86(\mathrm{~d}, 2 \mathrm{H}, J=8.4 \mathrm{~Hz}, \mathrm{Ar}-\mathrm{H}), 7.25$ (d, $2 \mathrm{H}, J=8.4 \mathrm{~Hz}, \mathrm{Ar}-\mathrm{H}), 7.33$ (s, 1H, Thiazole).

$\mathrm{N}$-(4-Methylbenzyl)- $\mathbf{N}$-((2-chlorothiazol-5-yl)methyl)-2-(methylthio) ethaneamine (15h). Yield, 81\%, colorless oil. ${ }^{1} \mathrm{H} \mathrm{NMR}\left(\mathrm{CDCl}_{3}, \delta(\mathrm{ppm})\right): 2.04\left(\mathrm{~s}, 3 \mathrm{H}, \mathrm{CH}_{3}\right), 2.19\left(\mathrm{~s}, 3 \mathrm{H}, \mathrm{CH}_{3}\right)$, 2.61-2.72 (m, 4H, $\left.\mathrm{CH}_{2}\right), 3.60$ (s, 2H, $\left.\mathrm{CH}_{2}\right), 3.71$ (s, 2H, $\left.\mathrm{CH}_{2}\right), 7.26-7.34$ (m, 5H, Ar-H).

$\mathrm{N}$-(4-Bromo-2-fluorobenzyl)- $\mathrm{N}$-((2-chlorothiazol-5-yl)methyl)-2-(methylthio) ethanamine (15i). Yield, 77\%, colorless oil. ${ }^{1} \mathrm{H} \mathrm{NMR}\left(\mathrm{CDCl}_{3}, \delta(\mathrm{ppm})\right)$ : 2.05 (s, 3H, $\left.\mathrm{CH}_{3}\right), 2.62-2.75(\mathrm{~m}, 4 \mathrm{H}$, $\left.\mathrm{CH}_{2}\right), 3.66$ (s, 2H, $\left.\mathrm{CH}_{2}\right), 3.77$ (s, 2H, $\left.\mathrm{CH}_{2}\right)$, 7.08-7.36 (m, 4H, Ar-H).

General procedure for the preparation of $N$-cyano 2 -(substituted amino)ethyl methyl sulfilimine (16)

To a solution of sulfide (1.0 mmol), $\mathrm{H}_{2} \mathrm{NCN}(55.0 \mathrm{mg}, 1.3 \mathrm{mmol})$ and $t$-BuOK (135.0 mg, 1.2 $\mathrm{mmol})$ in methanol $(6 \mathrm{~mL})$ at room temperature, NBS $(267.0 \mathrm{mg}, 1.5 \mathrm{mmol})$ was added. Once the starting material was consumed (monitored by TLC), the reaction mixture was concentrated under reduced pressure, saturated aqueous $\mathrm{Na}_{2} \mathrm{~S}_{2} \mathrm{O}_{3}$ was added and extracted with $\mathrm{CH}_{2} \mathrm{Cl}_{2}(3 \times 5$ $\mathrm{mL}$ ). The organic layer was dried over anhydrous magnesium sulfate, filtered and evaporated. The residue was purified by silica gel column chromatography (ethyl acetate-ethanol, 10:1) to afford the title compounds as colorless oil.

$\mathbf{N}$-(Cyano) 2-((benzyl) ((2-chlorothiazol-5-yl)methyl)amino)ethyl methyl sulfilimine (16a). Yield, 69\%, colorless oil. ${ }^{1} \mathrm{H} \mathrm{NMR}\left(\mathrm{CDCl}_{3}, \delta(\mathrm{ppm})\right): 2.63\left(\mathrm{~s}, 3 \mathrm{H}, \mathrm{CH}_{3}\right), 2.87-2.93\left(\mathrm{~m}, 2 \mathrm{H}, \mathrm{CH}_{2}\right)$, 3.06-3.23 (m, 2H, $\left.\mathrm{CH}_{2}\right), 3.54-3.78\left(\mathrm{~m}, 2 \mathrm{H}, \mathrm{CH}_{2}\right), 3.86\left(\mathrm{~d}, 2 \mathrm{H}, J=3.0 \mathrm{~Hz}, \mathrm{CH}_{2}\right), 7.28-7.39(\mathrm{~m}$, $6 \mathrm{H}, \mathrm{Ar}-\mathrm{H})$.

N-(Cyano) 2-((2-chlorobenzyl)((2-chlorothiazol-5-yl)methyl)amino)ethyl methyl sulfilimine (17b). Yield, 72\%, colorless oil. ${ }^{1} \mathrm{H}$ NMR $\left(\mathrm{CDCl}_{3}, \delta(\mathrm{ppm})\right): 2.64\left(\mathrm{~s}, 3 \mathrm{H}, \mathrm{CH}_{3}\right), 2.87-2.97(\mathrm{~m}, 2 \mathrm{H}$, $\left.\mathrm{CH}_{2}\right), 3.08-3.28\left(\mathrm{~m}, 2 \mathrm{H}, \mathrm{CH}_{2}\right), 3.81\left(\mathrm{~d}, 2 \mathrm{H}, J=3.3 \mathrm{~Hz}, \mathrm{CH}_{2}\right), 3.92\left(\mathrm{~s}, 2 \mathrm{H}, \mathrm{CH}_{2}\right), 7.26-7.29(\mathrm{~m}$, $3 \mathrm{H}, \mathrm{Ar}-\mathrm{H}), 7.38-7.41$ (m, 2H, Ar-H).

$\mathbf{N}$-(Cyano) 2-((2-fluorobenzyl)((2-chlorothiazol-5-yl)methyl)amino)ethyl methyl sulfilimine (16c).Yield, 69\%, colorless oil. ${ }^{1} \mathrm{H} \mathrm{NMR}\left(\mathrm{CDCl}_{3}, \delta\right.$ (ppm)): $2.67\left(\mathrm{~s}, 3 \mathrm{H}, \mathrm{CH}_{3}\right), 2.88-2.97(\mathrm{~m}, 2 \mathrm{H}$, $\left.\mathrm{CH}_{2}\right), 3.09-3.28\left(\mathrm{~m}, 2 \mathrm{H}, \mathrm{CH}_{2}\right), 3.56-3.82\left(\mathrm{~m}, 2 \mathrm{H}, \mathrm{CH}_{2}\right), 3.87\left(\mathrm{~d}, 2 \mathrm{H}, J=2.4 \mathrm{~Hz}, \mathrm{CH}_{2}\right), 6.98-7.11$ (m, 4H, Ar-H). 7.29-7.37 (m, 1H, Ar-H), 7.39 (s, 1H, Thiazole).

$\mathbf{N}$-(Cyano) 2-((3-fluorobenzyl)((2-chlorothiazol-5-yl)methyl)amino)ethyl methyl sulfilimine (16d). Yield, 70\%, colorless oil. ${ }^{1} \mathrm{H}$ NMR $\left(\mathrm{CDCl}_{3}, \delta(\mathrm{ppm})\right): 2.68\left(\mathrm{~s}, 3 \mathrm{H}, \mathrm{CH}_{3}\right), 2.90-2.97$ (m, $\left.2 \mathrm{H}, \mathrm{CH}_{2}\right), 3.08-3.26\left(\mathrm{~m}, 2 \mathrm{H}, \mathrm{CH}_{2}\right), 3.60-3.82\left(\mathrm{~m}, 2 \mathrm{H}, \mathrm{CH}_{2}\right), 3.87$ (d, 2H, J=2.4 Hz, $\mathrm{CH}_{2}$ ), 6.997.12 (m, 3H, Ar-H), 7.28-7.34 (m, 1H, Ar-H), 7.39 (s, 1H, Thiazole).

$\mathrm{N}$-(Cyano) 2-((4-fluorobenzyl)amino)((2-chlorothiazol-5-yl)methyl)ethyl methyl sulfilimine (16e). Yield, 75\%, colorless oil. ${ }^{1} \mathrm{H}$ NMR $\left(\mathrm{CDCl}_{3}, \delta(\mathrm{ppm})\right): 2.66\left(\mathrm{~s}, 3 \mathrm{H}, \mathrm{CH}_{3}\right), 2.86-2.96(\mathrm{~m}, 2 \mathrm{H}$, $\left.\mathrm{CH}_{2}\right), 3.05-3.27\left(\mathrm{~m}, 2 \mathrm{H}, \mathrm{CH}_{2}\right), 3.55-3.75\left(\mathrm{~m}, 2 \mathrm{H}, \mathrm{CH}_{2}\right), 3.85\left(\mathrm{~d}, J=2.4 \mathrm{~Hz}, 2 \mathrm{H}, \mathrm{CH}_{2}\right), 7.02-7.08$ (m, 2H, Ar-H), 7.27-7.32 (m, 2H, Ar-H), 7.39 (s, 1H, Thiazole).

$\mathbf{N}$-(Cyano) 2-((4-chlorobenzyl)((2-chlorothiazol-5-yl)methyl)amino)ethyl methyl sulfilimine (16f). Yield, 78\%, colorless oil. ${ }^{1} \mathrm{H}$ NMR $\left(\mathrm{CDCl}_{3}, \delta\right.$ (ppm)): 2.67 (s, 3H, $\left.\mathrm{CH}_{3}\right), 2.87-2.94(\mathrm{~m}, 2 \mathrm{H}$, 
$\left.\mathrm{CH}_{2}\right), 3.08-3.28\left(\mathrm{~m}, 2 \mathrm{H}, \mathrm{CH}_{2}\right), 3.56-3.74\left(\mathrm{~m}, 2 \mathrm{H}, \mathrm{CH}_{2}\right), 3.85\left(\mathrm{~d}, 2 \mathrm{H}, J=2.4 \mathrm{~Hz}, \mathrm{CH}_{2}\right), 7.25-7.38$ $(\mathrm{m}, 5 \mathrm{H}, \mathrm{Ar}-\mathrm{H})$.

$\mathrm{N}$-(Cyano) 2-((4-methoxybenzyl)((2-chlorothiazol-5-yl)methyl)amino)ethyl methyl sulfilimine (16g). Yield, 70\%, colorless oil. ${ }^{1} \mathrm{H} \mathrm{NMR}\left(\mathrm{CDCl}_{3}, \delta(\mathrm{ppm})\right)$ : $2.61\left(\mathrm{~s}, 3 \mathrm{H}, \mathrm{CH}_{3}\right), 2.87$ $2.94\left(\mathrm{~m}, 2 \mathrm{H}, \mathrm{CH}_{2}\right), 3.01-3.26\left(\mathrm{~m}, 2 \mathrm{H}, \mathrm{CH}_{2}\right), 3.6-3.74\left(\mathrm{~m}, 2 \mathrm{H}, \mathrm{CH}_{2}\right), 3.80$ (s, 3H, $\left.\mathrm{OCH}_{3}\right), 3.85$ (d, $\left.2 \mathrm{H}, J=3.3 \mathrm{~Hz}, \mathrm{CH}_{2}\right), 6.88$ (d, 2H, $\left.J=8.4 \mathrm{~Hz}, \mathrm{Ar}-\mathrm{H}\right), 7.21$ (d, 2H, J=8.4 Hz, Ar-H), 7.39 (s, 1H, Thiazole)..

$\mathbf{N}$-(Cyano) 2-((4-methylbenzyl) ((2-chlorothiazol-5-yl)methyl)amino)ethyl methyl sulfilimine (16h). Yield, 68\%, colorless oil. ${ }^{1} \mathrm{H} \mathrm{NMR}\left(\mathrm{CDCl}_{3}, \delta(\mathrm{ppm})\right): 2.34\left(\mathrm{~s}, 3 \mathrm{H}, \mathrm{CH}_{3}\right), 2.58$ (s, 3H, $\mathrm{CH}_{3}$ ), 2.67-2.93 (m, 2H, $\mathrm{CH}_{2}$ ), 3.05-3.25 (m, 2H, $\left.\mathrm{CH}_{2}\right), 3.50-3.85$ (m, 2H, $\mathrm{CH}_{2}$ ), 3.87 (d, $\left.2 \mathrm{H}, J=2.1 \mathrm{~Hz}, \mathrm{CH}_{2}\right), 7.17-7.28(\mathrm{~m}, 4 \mathrm{H}, \mathrm{Ar}-\mathrm{H}), 7.39$ (s, 1H, Thiazole).

N-(Cyano) 2-((2-fluoro-4-bromobenzyl) ((2-chlorothiazol-5-yl)methyl)amino)ethyl methyl sulfilimine (16i). Yield, 73\%, colorless oil. ${ }^{1} \mathrm{H} \mathrm{NMR}\left(\mathrm{CDCl}_{3}, \delta(\mathrm{ppm})\right): 2.74\left(\mathrm{~s}, 3 \mathrm{H}, \mathrm{CH}_{3}\right), 2.85$ $3.08\left(\mathrm{~m}, 3 \mathrm{H}, \mathrm{CH}_{2}\right), 3.21-3.28\left(\mathrm{~m}, 1 \mathrm{H}, \mathrm{CH}_{2}\right), 3.72\left(\mathrm{~s}, 2 \mathrm{H}, \mathrm{CH}_{2}\right), 3.86\left(\mathrm{~s}, 2 \mathrm{H}, \mathrm{CH}_{2}\right), 7.24-7.34(\mathrm{~m}$, $3 \mathrm{H}, \mathrm{Ar}-\mathrm{H}), 7.40$ (s, 1H, Thiazole).

General procedure for the preparation of $\mathrm{N}$-cyano 2 -(substituted amino)ethyl methyl sulfoximine (17)

To a stirred solution 3-chloroperbenzoic acid $(0.81 \mathrm{mg}, 4 \mathrm{mmol})$ in ethanol $(5 \mathrm{~mL})$ cooled to 0 was added a solution of potassium carbonate $(0.82 \mathrm{mg}, 6 \mathrm{mmol})$ in water $(4 \mathrm{~mL})$. The resulting mixture was stirred a 0 for $20 \mathrm{~min}$. Then the solution of the sulfilimine starting material (2 $\mathrm{mmol})$ in ethanol $(4 \mathrm{~mL})$ was added at once. The resulting mixture was stirred for 20 min at 0 and saturated sodium bisulfite was added to quench the excess peracid. The resulting mixture was extracted with dichloromethane and dried over anhydrous $\mathrm{Na}_{2} \mathrm{SO}_{4}$. The solvent was removed under reduce pressure and the residue was purified by column chromatography (ethyl acetate-ethanol, 10:1) to afford the title compounds.

$\mathrm{N}$-(Cyano) 2-((benzyl)((2-chlorothiazol-5-yl)methyl)amino)ethyl methyl sulfoximine (17a). Yield, 65\%, white crystalline solid, mp 80-82 ${ }^{\circ} \mathrm{C}$. IR $\left(\mathrm{KBr}, \mathrm{cm}^{-1}\right)$ v: $2198(\mathrm{CN}) .{ }^{1} \mathrm{H}$ NMR $\left(\mathrm{CDCl}_{3}, \delta(\mathrm{ppm})\right): 3.10-3.17\left(\mathrm{~m}, 2 \mathrm{H}, \mathrm{CH}_{2}\right), 3.20\left(\mathrm{~s}, 3 \mathrm{H}, \mathrm{CH}_{3}\right), 3.37-3.43\left(\mathrm{~m}, 2 \mathrm{H}, \mathrm{CH}_{2}\right), 3.67$ (s, $\left.2 \mathrm{H}, \mathrm{CH}_{2}\right), 3.85\left(\mathrm{~s}, 2 \mathrm{H}, \mathrm{CH}_{2}\right), 7.27-7.37(\mathrm{~m}, 5 \mathrm{H}, \mathrm{Ar}-\mathrm{H}), 7.41\left(\mathrm{~s}, 1 \mathrm{H}\right.$, Thiazole). ${ }^{13} \mathrm{C}$ NMR $\left(100 \mathrm{MHz}, \mathrm{CDCl}_{3}\right) \delta(\mathrm{ppm}): 41.59,47.18,50.58,52.89,58.18,111.87(\mathrm{CN}), 128.20,128.90$, 129.07, 136.52, 137.89, 140.11, 152.22. Anal. Calcd for $\mathrm{C}_{15} \mathrm{H}_{17} \mathrm{ClN}_{4} \mathrm{OS}_{2}: \mathrm{C}, 48.84 ; \mathrm{H}, 4.64 ; \mathrm{N}$, 15.19; Found: C, 48,70; H, 4.59; N, 15.20.

$\mathrm{N}$-(Cyano) 2-((2-chlorobenzyl)((2-chlorothiazol-5-yl)methyl)amino)ethyl methyl sulfoximine (17b). Yield, 70\%, white crystalline solid, mp 100-102 ${ }^{\circ} \mathrm{C}$. IR $\left(\mathrm{KBr}, \mathrm{cm}^{-1}\right)$ v: 2200 $(\mathrm{CN}) .{ }^{1} \mathrm{H} \mathrm{NMR}\left(\mathrm{CDCl}_{3}, \delta(\mathrm{ppm})\right)$ : 3.13-3.24 (m, 5H), 3.32-3.45 (m, 2H, $\left.\mathrm{CH}_{2}\right), 3.80\left(\mathrm{~s}, 2 \mathrm{H}, \mathrm{CH}_{2}\right)$, $3.93\left(\mathrm{~s}, 2 \mathrm{H}, \mathrm{CH}_{2}\right), 7.28-7.40(\mathrm{~m}, 4 \mathrm{H}, \mathrm{Ar}-\mathrm{H}), 7.43$ (s, $1 \mathrm{H}$, Thiazole). ${ }^{13} \mathrm{C} \mathrm{NMR}\left(100 \mathrm{MHz}, \mathrm{CDCl}_{3}\right)$ $\delta(\mathrm{ppm}): 41.62,47.07,50.87,52.82,55.61,111.79(\mathrm{CN}), 127.27,129.61,130.13,131.23,134.36$, 134.48, 137.42, 140.30. 152.30. Anal. Calcd for $\mathrm{C}_{15} \mathrm{H}_{16} \mathrm{Cl}_{2} \mathrm{~N}_{4} \mathrm{OS}_{2}$ : C, 44.67; H, 4.00; N, 13.89; Found: C, 44.77; H, 3.95; N, 13.71 . 
$N$-(Cyano) 2-((2-fluorobenzyl) ((2-chlorothiazol-5-yl)methyl)amino)ethyl methyl sulfoximine (17c). Yield, 62\%, white crystalline solid, mp 89-90 ${ }^{\circ} \mathrm{C}$. IR $\left(\mathrm{KBr}, \mathrm{cm}^{-1}\right)$ v: 2190 $(\mathrm{CN}) .{ }^{1} \mathrm{H}$ NMR $\left(\mathrm{CDCl}_{3}, \delta(\mathrm{ppm})\right)$ : 3.11-3.18 (m, 2H, $\left.\mathrm{CH}_{2}\right), 3.27\left(\mathrm{~s}, 3 \mathrm{H}, \mathrm{CH}_{3}\right), 3.42-3.49(\mathrm{~m}, 2 \mathrm{H}$, $\left.\mathrm{CH}_{2}\right), 3.74\left(\mathrm{~s}, 2 \mathrm{H}, \mathrm{CH}_{2}\right), 3.88\left(\mathrm{~s}, 2 \mathrm{H}, \mathrm{CH}_{2}\right), 7.07-7.40(\mathrm{~m}, 4 \mathrm{H}, \mathrm{Ar}-\mathrm{H}), 7.43$ (s, $1 \mathrm{H}$, Thiazole). ${ }^{13} \mathrm{C}$ NMR (100MHz, $\left.\mathrm{CDCl}_{3}\right) \delta(\mathrm{ppm}): 41.79,47.14,50.47,50.95,52.76,112.03(\mathrm{CN}), 115.70,115.98$, $123.01,123.20,124.52$, 124.57, 130.20, 131.70, 137.74, 140.22, 152.19, 159.77. Anal. Calcd for $\mathrm{C}_{15} \mathrm{H}_{16} \mathrm{ClFN}_{4} \mathrm{OS}_{2}$ : C, 46.57; H, 4.17; N, 14.48; Found: C, 46.45; H, 4,38; N, 14.50 .

$\mathrm{N}$-(Cyano) 2-((3-fluorobenzyl) ((2-chlorothiazol-5-yl)methyl)amino)ethyl methyl sulfoximine (17d). Yield, $65 \%$, white crystalline solid, mp 73-74 ${ }^{\circ} \mathrm{C}$. IR $\left(\mathrm{KBr}, \mathrm{cm}^{-1}\right)$ v: 2198 $(\mathrm{CN}) .{ }^{1} \mathrm{H} \mathrm{NMR}\left(\mathrm{CDCl}_{3}, \delta(\mathrm{ppm})\right):{ }^{1} 3.13-3.19\left(\mathrm{~m}, 2 \mathrm{H}, \mathrm{CH}_{2}\right), 3.23\left(\mathrm{~s}, 3 \mathrm{H}, \mathrm{CH}_{3}\right), 3.43-3.49(\mathrm{~m}, 2 \mathrm{H}$, $\mathrm{CH}_{2}$ ), 3.68 (s, 2H, $\mathrm{CH}_{2}$ ), 3.86 (s, 2H, $\left.\mathrm{CH}_{2}\right), 7.0-7.09$ (m, 3H, Ar-H), 7.26-7.38 (m, 1H, Ar-H), 7.42 (s, 1H, Thiazole). ${ }^{3} \mathrm{C}$ NMR $\left(100 \mathrm{MHz}, \mathrm{CDCl}_{3}\right) \delta(\mathrm{ppm}): 41.63,46.96,50.38,52.81,57.58$, $111.73(\mathrm{CN}), 115.01,115.55,115.83,124.50,124.52,130.44,130.49,130.55,137.51,139.29$, 140.21, 152.40, 161.42. Anal. Calcd for $\mathrm{C}_{15} \mathrm{H}_{16} \mathrm{ClFN}_{4} \mathrm{OS}_{2}$ : C, 46.57; H, 4.17; N, 14.48; Found: C, $46.45 ; \mathrm{H}, 4.21 ; \mathrm{N}, 14.29$.

$\mathrm{N}$-(Cyano) 2-((4-fluorobenzyl)((2-chlorothiazol-5-yl)methyl)amino)ethyl methyl sulfoximine (17e). Yield, $75 \%$, white crystalline solid, mp 90-91 ${ }^{\circ} \mathrm{C}$. IR $\left(\mathrm{KBr}, \mathrm{cm}^{-1}\right)$ v: $2195(\mathrm{CN}) .{ }^{1} \mathrm{H}$ NMR $\left(\mathrm{CDCl}_{3}, \delta(\mathrm{ppm})\right): 3.11-3.18\left(\mathrm{~m}, 2 \mathrm{H}, \mathrm{CH}_{2}\right), 3.22\left(\mathrm{~s}, 3 \mathrm{H}, \mathrm{CH}_{3}\right), 3.38-3.44\left(\mathrm{~m}, 2 \mathrm{H}, \mathrm{CH}_{2}\right), 3.65(\mathrm{~s}$, 2H, $\left.\mathrm{CH}_{2}\right), 3.85\left(\mathrm{~s}, 2 \mathrm{H}, \mathrm{CH}_{2}\right), 7.04-7.09$ (m, 2H, Ar-H), 7.24--7.29 (m, 2H, Ar-H), 7.41 (s, 1H, Thiazole). ${ }^{13} \mathrm{C}$ NMR $\left(100 \mathrm{MHz}, \mathrm{CDCl}_{3}\right) \delta(\mathrm{ppm}): 41.63,46.78,50.14,52.69,57.25,111.92(\mathrm{CN})$, $115.64,115.92,130.57,130.68,132.32,132.34,137.78,140.12,152.24,160.81$. Anal. Calcd for $\mathrm{C}_{15} \mathrm{H}_{16} \mathrm{ClFN}_{4} \mathrm{OS}_{2}: \mathrm{C}, 46.57 ; \mathrm{H}, 4.17 ; \mathrm{N}, 14.48$; Found: C, 46.65; H, 4.10; N, 14.35

$\mathrm{N}$-(Cyano) 2-((4-chlorobenzyl)((2-chlorothiazol-5-yl)methyl)amino)ethyl

methyl sulfoximine (17f). Yield, 72\%, white crystalline solid, mp 98-100 ${ }^{\circ} \mathrm{C}$. IR $\left(\mathrm{KBr}, \mathrm{cm}^{-1}\right)$ v: 2190 $(\mathrm{CN}) .{ }^{1} \mathrm{H} \mathrm{NMR}\left(\mathrm{CDCl}_{3}, \delta(\mathrm{ppm})\right)$ : 3.09-3.18 (m, 2H, $\left.\mathrm{CH}_{2}\right), 3.22\left(\mathrm{~s}, 3 \mathrm{H}, \mathrm{CH}_{3}\right), 3.33-3.48(\mathrm{~m}, 2 \mathrm{H}$, $\left.\mathrm{CH}_{2}\right), 3.65$ (s, 2H, $\left.\mathrm{CH}_{2}\right), 3.84\left(\mathrm{~s}, 2 \mathrm{H}, \mathrm{CH}_{2}\right), 7.22$ (d, 2H, Ar-H), 7.35 (d, 2H, Ar-H), 7.40 (s, 1H, Thiazole). ${ }^{13} \mathrm{C}$ NMR $\left(100 \mathrm{MHz}, \mathrm{CDCl}_{3}\right) \delta(\mathrm{ppm}): 41.54,46.63,49.91,52.45,57.08,111.88(\mathrm{CN})$, 128.90, 130.19,133.73, 135.03, 137.59, 140.03, 152.13. MS (EI), m/z $427.41\left([\mathrm{M}+23+1]^{+}, 90\right)$, $425.44\left([\mathrm{M}+23-1]^{+}, 100\right)$. Anal. Calcd for $\mathrm{C}_{15} \mathrm{H}_{16} \mathrm{Cl}_{2} \mathrm{~N}_{4} \mathrm{OS}_{2}: \mathrm{C}$, 44.67; H, 4.00; N, 13.89; Found: C, 44.62; H, 4.10; N, 13.80 .

N-(Cyano)2-((4-methoxybenzyl)((2-chlorothiazol-5-yl)methyl)amino)ethyl methyl sulfoximine (17g). Yield, 68\%, colorless oil. IR (KBr, cm-1) v: $2200(\mathrm{CN}) .{ }^{1} \mathrm{H}$ NMR (CDCl3, $\delta$ (ppm)): 3.08-3.15 (m, 2H, $\left.\mathrm{CH}_{2}\right), 3.21\left(\mathrm{~s}, 3 \mathrm{H}, \mathrm{CH}_{3}\right), 3.29-3.44\left(\mathrm{~m}, 2 \mathrm{H}, \mathrm{CH}_{2}\right), 3.62\left(\mathrm{~s}, 2 \mathrm{H}, \mathrm{CH}_{2}\right)$, $3.82\left(\mathrm{~s}, 3 \mathrm{H}, \mathrm{OCH}_{3}\right), 3.87\left(\mathrm{~s}, 2 \mathrm{H}, \mathrm{CH}_{2}\right), 6.90(\mathrm{~d}, 2 \mathrm{H}, \mathrm{Ar}-\mathrm{H}), 7.19$ (d, 2H, Ar-H), $7.41(\mathrm{~s}, 1 \mathrm{H}$, Thiazole). ${ }^{13} \mathrm{C}$ NMR $\left(100 \mathrm{MHz}, \mathrm{CDCl}_{3}\right) \delta(\mathrm{ppm}): 41.65,47.06,50.45,52.87,55.32,57.53,111.91$ $(\mathrm{CN}), \quad 114.29, \quad 128.15, \quad 130.35,137.79,140.14,152.24,159.52$. Anal. Calcd for: C16H19CIN4O2S2: C, 48.17; H, 4.80; N, 14.04; Found: C, 48.30; H, 4.89; N, 14.00.

$\mathrm{N}$-(Cyano) 2-((4-methylbenzyl)((2-chlorothiazol-5-yl)methyl)amino)ethyl methyl sulfoximine (17h). Yield, $72 \%$, white crystalline solid, mp 85-87 ${ }^{\circ} \mathrm{C}$. IR (KBr, cm $\left.{ }^{-1}\right)$ v: 2198 $(\mathrm{CN}) .{ }^{1} \mathrm{H} \mathrm{NMR}\left(\mathrm{CDCl}_{3}, \delta(\mathrm{ppm})\right): 2.35\left(\mathrm{~s}, 3 \mathrm{H}, \mathrm{CH}_{3}\right), 3.09-3.16\left(\mathrm{~m}, 2 \mathrm{H}, \mathrm{CH}_{2}\right), 3.21\left(\mathrm{~s}, 3 \mathrm{H}, \mathrm{CH}_{3}\right)$, 
3.26-3.42 (m, 2H, $\left.\mathrm{CH}_{2}\right), 3.62\left(\mathrm{~s}, 2 \mathrm{H}, \mathrm{CH}_{2}\right), 3.84\left(\mathrm{~s}, 2 \mathrm{H}, \mathrm{CH}_{2}\right), 7.13-7.18(\mathrm{~m}, 4 \mathrm{H}, \mathrm{Ar}-\mathrm{H}), 7.40$ (s, $1 \mathrm{H}$, Thiazole). ${ }^{13} \mathrm{C} \mathrm{NMR}\left(100 \mathrm{MHz}, \mathrm{CDCl}_{3}\right) \delta(\mathrm{ppm}): 21.12,41.65,47.32,50.72,53.03,57.95$, $111.78(\mathrm{CN}), 129.04,129.39,133.26,137.81,138.06,140.09,152.24$. Anal. Calcd for $\mathrm{C}_{16} \mathrm{H}_{19} \mathrm{ClN}_{4} \mathrm{OS}_{2}$ : C, 50.18; H, 5.00; N, 14.63; Found: C, 50.30; H, 5.09; N, 14.45.

$\mathrm{N}$-(Cyano) 2-((2-fluoro-4-bromobenzyl)((2-chlorothiazol-5-yl)methyl)amino)ethyl methyl sulfoximine (17i). Yield, 69\%, white crystalline solid, mp 103-104 ${ }^{\circ} \mathrm{C}$. IR $\left(\mathrm{KBr}, \mathrm{cm}^{-1}\right)$ v: 2190 $(\mathrm{CN}) .{ }^{1} \mathrm{H} \mathrm{NMR}\left(\mathrm{CDCl}_{3}, \delta(\mathrm{ppm})\right)$ : 3.10-3.19 (m, 2H, $\left.\mathrm{CH}_{2}\right), 3.21\left(\mathrm{~s}, 3 \mathrm{H}, \mathrm{CH}_{3}\right), 3.27-3.49(\mathrm{~m}, 2 \mathrm{H}$, $\mathrm{CH}_{2}$ ), 3.70 (s, 2H, $\mathrm{CH}_{2}$ ), 3.85 (s, 2H, $\left.\mathrm{CH}_{2}\right), 7.13-7.19$ (m, 1H, Ar-H), 7.26-7.34 (m, 2H, Ar-H), 7.42 (s, 1H, Thiazole). ${ }^{13} \mathrm{C}$ NMR $\left(100 \mathrm{MHz}, \mathrm{CDCl}_{3}\right) \delta(\mathrm{ppm}): 41.86,46.95,50.29,50.52,52.71$, $111.87(\mathrm{CN}), 119.55,122.27,122.47,127.93,132.59,137.38,140.29,152.39$, 159.45. Anal. Calcd for $\mathrm{C}_{15} \mathrm{H}_{15} \mathrm{BrClFN}_{4} \mathrm{OS}_{2}$ : C, 38.68; H, 3.25; N, 12.03; Found: C, 38.59; H, 3.24; N, 12.00 .

\section{Biology assay}

The bioassay was performed on a representative test organism reared in the laboratory. The bioassay was repeated at $25 \pm 1$ according to statistical requirements. Assessments were made on a dead/alive basis, and mortality rates were corrected using Abbott's formula. Evaluations are based on a percentage scale of $0-100$ in which $0=$ no activity and $100=$ total kill.

The insecticidal activities of the title compounds were tested against Aphis laburni Kaltenbach by foliar application. About 60 aphids were transferred to the shoot with 3-5 fresh leaves of horsebean. The shoot with aphids was cut and dipped into the solution of $10 \mathrm{mg} / \mathrm{L}$ of test compound for $2 \mathrm{~s}$, after removing extra solutions on the leaf; the aphids were raised in the shoot at $25 \pm 1$ and $85 \%$ relative humidity for $16 \mathrm{~h}$. Each experiment for one compound was triplicated. The revised death rate was calculated by Abbott's formula.

\section{Acknowledgements}

We are grateful to Dr Bin Li for providing us with invaluable suggestions. We appreciate the excellent data on biological activities measured by Mr Hong Zhang and Mrs Yanmei Luo. We gratefully acknowledge the National Natural Science Foundation of China (NNSFC) (No. 20772068) and the National Key Project of Scientific and Technical Supporting Programs Funded by Ministry of Science \& Technology of China (NO. 2006BAE01A01-5) for financial support.

\section{References}

1. Shiokawa, K.; Tsuboi, S.; Kagabu, S.; Moriya, K. JP. Pat. 18 627, 1985; Chem. Abstr. 1987, $106,28848$. 
2. Minamida, I.; Iwanaga, K.; Tabuchi, T.; Aoki, I.; Fusaka, T.; Ishizuka, H.; Okauchi,; T. J. Pestic. Sci. 1993, 18, 41.

3. Takahashi, H.; Mitsui, J.; Takakusa, N.; Matsuda, M. Yoneda, H.; Suzuki, J.; Ishimitsu, K.; Kishimoto, T. Proc Brighton CropProtect Conf_Pests and Diseases 1992, 89.

4. Shiokawa, K.; Tsuboi, S.; Kagabu, S.; Sasaki, S.; Moriya, K.; Hattori, Y. Eur. Pat. Appl. 235 725, 1987; Chem. Abstr. 1988, 108, 21897.

5. Senn. R.; Hofer, D. Hoppe, T.; Angst, M.; Wyss, P.; Brandl, F. Maienfisch, P.; Zang, L.; White, S. Proc Brighton Crop Protect Conf-Pest and Diseases 1998, 27.

6. Maienfisch, P.; Huerlimann, H.; Rindlisbacher, A.; Gsell, L.; Dettwiler, H.; Haettenschwiler, J.; Sieger, E.; Walti, M. Pest Manag. Sci. 2001, 57, 165.

7. Uneme, H.; Iwanaga, K.; Higuchi, N.; Minamida, I.; Okauchi, T. Eur. Pat. Appl. 376 279, 1990; Chem. Abstr. 1990, 114, 61934.

8. Wakita, T.; Kinoshita, K.;Yamada, E.; Yasui, N.; Kawahara, N.; Naoi, A.; Nakaya, M.; Ebihara, K.; Matsuno, H.; Kodaka, K. Pestic. Sci. 2000, 59, 1066.

9. Bai, D.; Lummis, S. C. R.; Leicht, W.; Breer, H.; Sattelle, D. B. Pestic. Sci. 1991, 33, 197.

10. Liu, M.; Casida, J. E. Pestic. Biochem. Physiol. 1993, 46, 40.

11. Nishimura, K.; Kanda, Y.; Okazawa, A.; Ueno, T. Pestic. Biochem. Physiol. 1994, 50, 51.

12. Mori, K.; Okumoto, T.; Kawahara, N.; Ozoe, Y. Pest Manag. Sci. 2001, 58, 190-196.

13. Elbert, A.; Nauen, R. Pest Manage. Sci. 2000, 56, 60.

14. Rauch, N; Nauen, R. Arch. Insects Biochem. Physiol. 2003, 54, 165.

15. Nauen, R; Denholm, I. Arch. Ins. Biochem. Physiol. 2005, 58, 200.

16. Loso, M. R.; Nugent, B. M.; Huang, J. X.; Rogers, R. B.; Zhu, Y. M.; Renga, J.M.; Hegde, V. B.; Demark, J. J.. PCT. Int. Appl. 2007095229, 2007; Chem. Abstr. 2008, 147, 270793.

17. Loso, M. R.; Nugent, B. M.; Huang, J. X. Insecticidal $N$-substituted (heteroaryl) cycloalkyl sulfoximines. PCT Int. Appl. 2008027073, 2008; Chem. Abstr. 2008, 148, 331556.

18. Zhu, Y. M.; Loso, M. R.; Nugent, B. M.; Huang, J. X. Rogers, R. B. PCT Int. Appl. 2008057129, 2008; Chem. Abstr. 2008, 148, 511121.

19. Loso, M. R.; Nugent, B. M.; Zhu, Y. M.; Rogers, R. B.; Huang, J. X.; Renga, J. M.; Whiteker, G. T.; Breaux, N. T.; Daeuble, J. F. PCT Int. Appl. 2008057131, 2008; Chem. Abstr. 2008, 148, 511120.

20. Huang, J. X.; Rogers, R. B.; Orr, N.; Sparks, T. C.; Gifford, J. M.; Loso, M. R. Zhu, Y. M.; Meade, T. PCT Int. Appl. 2007149134, 2007; Chem. Abstr. 2007, 148, 47562.

21. Hillstrom, G. F.; Hockman, M. A.; Murugan, R..; Scriven, E. F. V. Jason, Y. ARKIVOC 2001, (vi), 94.

22. Bachir, L.; Kevin, D. A.; John, E. C. J. Med. Chem. 1999, 42, 2227.

23. Roush, D. M.; Chiarello, J. F.; Theodoridis, G.; Qi, H. G. PCT Int. Appl. 2006050121, 2006; Chem. Abstr. 2006, 144, 450623.

24. Mancheno, O. G.; Bistri, O.; Bolm, C. Org. Lett. 2007, 9, 3809. 